EPJ Web of Conferences 21, 08007 (2012)

DOI: $10.1051 /$ epjconf/20122108007

(c) Owned by the authors, published by EDP Sciences, 2012

\title{
Applications of Event-by-Event Fission Modeling with FREYA
}

\author{
R. Vogt ${ }^{1,2, a}$ and J. Randrup ${ }^{3}$ \\ 1 Physics Division, Lawrence Livermore National Laboratory, Livermore, CA 94551, USA \\ 2 Physics Department, University of California, Davis, CA 95616, USA \\ 3 Nuclear Science Division, Lawrence Berkeley National Laboratory, Berkeley, CA 94720, USA
}

\begin{abstract}
The recently developed code FREYA (Fission Reaction Event Yield Algorithm) generates large samples of complete fission events, consisting of two receding product nuclei as well as a number of neutrons and photons, all with complete kinematic information. Thus it is possible to calculate arbitrary correlation observables whose behavior may provide unique insight into the fission process. We first discuss the present status of FREYA, which has now been extended to include spontaneous fission. Concentrating on ${ }^{239} \mathrm{Pu}\left(\mathrm{n}_{\mathrm{th}}, \mathrm{f}\right),{ }^{240} \mathrm{Pu}(\mathrm{sf})$ and ${ }^{252} \mathrm{Cf}(\mathrm{sf})$, we discuss the neutron multiplicity correlations, the dependence of the neutron energy spectrum on the neutron multiplicity, and the relationship between the fragment kinetic energy and the number of neutrons and their energies. We also suggest novel fission observables that could be measured with modern detectors.
\end{abstract}

\section{Introduction}

Phenomenological studies of nuclear fission are of particular interest for possible practical applications in the fields of nonproliferation and security. In particular, the detection of special nuclear material (SNM) has risen in priority. Since all SNM emits neutrons, it is advantageous to use these neutron emissions for the detection of such material. For example, in highly enriched samples of plutonium $\left(90 \%{ }^{239} \mathrm{Pu}, 10 \%{ }^{240} \mathrm{Pu}\right)$ and uranium $\left(90 \%{ }^{235} \mathrm{U}, 10 \%{ }^{238} \mathrm{U}\right)$, the small content of ${ }^{240} \mathrm{Pu}$ and ${ }^{238} \mathrm{U}$ undergoes spontaneous fission, emitting on average two neutrons per fission. If it were possible to employ observable differences in the characteristics of the fission process between the two components of the material, it might be possible to distinguish between enriched and non-enriched samples of SNM. The penetrating nature of neutrons, together with their low background, give them intrinsic benefits over other observables [1].

Heretofore, most fission simulations have assumed that all emitted neutrons are drawn from the same energy spectrum which precludes correlations between the neutron mutliplicity and the associated spectral shape. In our event-by-event treatment, such inherent correlations are automatically included. Our approach employs the fission model FREYA (Fission Reaction Event Yield Algorithm) which incorporates the relevant physics with a few key parameters determined by comparison to data [2-4]. It simulates the entire fission process and produces complete fission events with full kinematic information on the emerging fission products and the emitted neutrons and photons, incorporating sequential neutron evaporation from the fission fragments. FREYA provides a means of using readilymeasured observables to improve our understanding of the fission process. Thus it is a potentially powerful tool for bridging the gap between current microscopic models and important fission observables as well as for improving estimates of the fission characteristics important for applications.

In this proceedings, based on a larger study [5], we compare and contrast correlations between neutron observables in spontaneous and thermal fission of ${ }^{240} \mathrm{Pu},{ }^{240} \mathrm{Pu}(\mathrm{sf})$ and ${ }^{239} \mathrm{Pu}\left(\mathrm{n}_{\mathrm{th}}, \mathrm{f}\right)$ respectively. We also study these same observables in the spontaneous fission of ${ }^{252} \mathrm{Cf}$, often used as a calibrator for

a e-mail: vogt2@llnl.gov

This is an Open Access article distributed under the terms of the Creative Commons Attribution-Noncommercial License 3.0, which permits unrestricted use, distribution, and reproduction in any noncommercial medium, provided the original work is properly cited. 
other fission measurements. We describe the experimental data on fission fragment mass distributions and the total fragment kinetic energy as a function of fragment mass that we employ. We then discuss various neutron observables, including the prompt fission neutron multiplicity as function of fragment mass, the neutron multiplicity distribution, and the energy spectrum of the prompt fission neutrons. We also study the neutron-neutron angular correlations as well as the correlations between both the total kinetic energy of the fission products and their residual excitation energy as a function of the total neutron multiplicity.

\section{FREYA Inputs}

The treatment of spontaneous fission in FREYA is similar to that of neutron-induced fission, except for the simplification that there is no pre-fission emission. Thus, generally, we start with a fissile nucleus ${ }^{A_{0}} Z_{0}$ with a specified excitation energy $E_{0}^{*}$, and let it undergo binary fission into a heavy fragment ${ }^{A_{H}} Z_{H}$ and a complementary light fragment ${ }^{A_{L}} Z_{L}$. The fragment masses are obtained from experimental mass yields by the procedure employed in the original description of FREYA [3].

The fragment mass yields, $Y(A)$, are assumed to exhibit three distinct modes of Gaussian form [7],

$$
Y(A)=S_{1}(A)+S_{2}(A)+S_{L}(A) .
$$

The first two terms represent asymmetric fission modes associated with the spherical shell closure at $N=82$ and the deformed shell closure at $N=88$, respectively, while the last term represents a broad symmetric mode. The asymmetric modes have a two-Gaussian form while the symmetric mode is given by a single Gaussian. Since each event leads to two fragments, the yields are normalized so that $\sum_{A} Y(A)=2$.

The results are shown for the fission fragment and the subsequent product yields on the left-hand side of Fig. 1. The yields reported for spontaneous fission are for the (primary) fragments while those reported for inducd fission are for the (post-evaporation) products [8]. The product yields are obtained after FREYA has finished emitting neutrons from the excited fragments. All the yields exhibit similar behavior, a rather broad double-humped distribution with a gap near symmetry, $A_{0} / 2$. The symmetric contribution is typically very small.

The plutonium results are closely related because both ${ }^{239} \mathrm{Pu}\left(\mathrm{n}_{\mathrm{th}}, \mathrm{f}\right)$ and ${ }^{240} \mathrm{Pu}(\mathrm{sf})$ start from a compound nucleus with the same value of $A_{0}$. The ${ }^{240} \mathrm{Pu}(\mathrm{sf})$ data were taken from a study of ${ }^{238,240,242} \mathrm{Pu}(\mathrm{sf})$ relative to ${ }^{239} \mathrm{Pu}\left(\mathrm{n}_{\mathrm{th}}, \mathrm{f}\right)$ [9]. The ${ }^{252} \mathrm{Cf}(\mathrm{sf})$ fragment yields are from an experiment [10] focused on the far asymmetric mass region.

There is a clear shift between the fragment yields (before neutron emission) and the product yields (after neutron emission). The magnitude of the shift depends on the overall mean neutron multiplicity, $v$, which in turn depends on the partition of the excitation energy between the light and heavy fragments. The shift is not symmetric but is larger for the light fragment, especially near symmetry. The location of the peak in the heavy fragment yield at $A \approx 130$ does not exhibit a significant shift due to neutron emission in any of the cases shown, even though the shift is apparent for other values of $A$. This is due to the proximity of the doubly-magic closed shell with $Z_{H}=50$ and $N_{H}=82$. This behavior is also apparent in the shape of $\operatorname{TKE}\left(A_{H}\right)$ and in the dependence of the mean neutron multiplicity on fragment mass, $v(A)$, as we soon discuss.

Once the partition of the total mass and charge between the two fragments has been selected, the $Q$ value of that particular fission channel follows from the difference between the total mass of the fissioning nucleus and the ground-state masses of the two fragments,

$$
Q_{L H}=M\left(A_{0}\right)-M_{L}-M_{H} .
$$

The $Q_{L H}$ value for the selected fission channel is then divided up between the total kinetic energy (TKE) and the total excitation energy (TXE) of the two fragments.

The right-hand side of Fig. 1 shows the measured average TKE as a function of the mass number of the heavy fragment, $A_{H}$. Near symmetry, the plutonium fission fragments are mid-shell nuclei subject to strong deformations. Thus the scission configuration will contain significant deformation energy and 

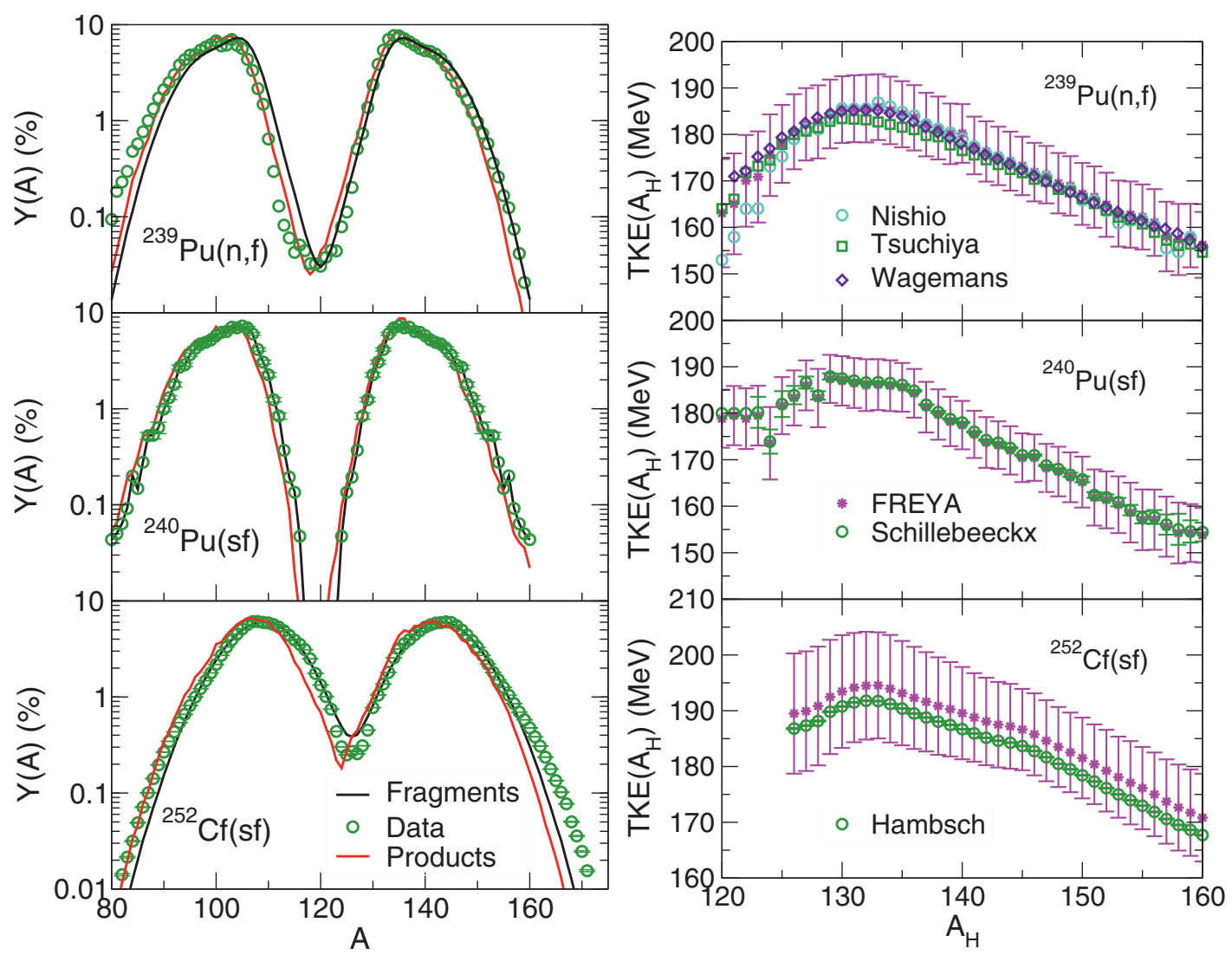

Fig. 1. (Left) The percent yield as a function of fragment mass for ${ }^{239} \mathrm{Pu}\left(\mathrm{n}_{\mathrm{th}}, \mathrm{f}\right)$ [8] (top), ${ }^{240} \mathrm{Pu}(\mathrm{sf})$ [9] (middle) and ${ }^{252} \mathrm{Cf}$ (sf) (bottom) [10]. The product yield data are shown for ${ }^{239} \mathrm{Pu}\left(\mathrm{n}_{\mathrm{th}}, \mathrm{f}\right)$ while the fragment yields are given for ${ }^{240} \mathrm{Pu}(\mathrm{sf})$ and ${ }^{252} \mathrm{Cf}(\mathrm{sf})$. The black curves are the 5-Gaussian fits to the fragment distributions while the red curves are the results after neutron emission in FREYA. (Right) The total fragment kinetic energies as a function of the heavy fragment mass for ${ }^{239} \mathrm{Pu}\left(n_{\mathrm{th}}, f\right)[12-14]$ (top), ${ }^{240} \mathrm{Pu}$ (sf) [9] (middle) and ${ }^{252} \mathrm{Cf}$ (sf) [10] (bottom). The FREYA results are shown with the calculated variance arising from the range of charges available for each $A_{H}$.

the TKE will be correspondingly low. At $A_{H}=132$, the heavy fragment is close to the doubly-magic closed shell with $Z_{H}=50$ and $N_{H}=82$ and is therefore resistant to distortions away from sphericity. Consequently, the scission configuration is fairly compact, and the TKE exhibits a maximum even though the light fragment is far from a closed shell and hence significantly deformed. Note that the peak around $A_{H}=132$ is a feature of all the data sets shown, regardless of whether fission is neutron induced or spontaneous and independent of the identity of the fissile nucleus.

The ${ }^{239} \mathrm{Pu}\left(\mathrm{n}_{\text {th }}, \mathrm{f}\right)$ data sets are very consistent for $A_{H}>135$, above the closed shell at $A_{H}=132$. In the region of the closed shell and below, the agreement among the data sets is not as good, particularly near the symmetry value of $A_{H}=120$, presumably due to the low fragment yields in this region. Unfortunately, no uncertainties are given on the data, only the full-width half maximum spread of TKE for several given values of $A_{H}$ in the measurment of Nishio et al. [12]. This variance is similar to that shown for FREYA. The ${ }^{240} \mathrm{Pu}$ (sf) data by Schillebeeckx et al. [9] are somewhat flatter in the region of the closed shell. There are considerable fluctuations in the data for $A_{H}<130$ and $\operatorname{TKE}\left(A_{H}\right)$ was not measured for $A_{H}<122$. We have therefore extrapolated a constant average value back to $A_{H}=$ 120. The ${ }^{252} \mathrm{Cf}(\mathrm{sf})$ data are again taken from Ref. [10] with $\cos \theta>0.9$. The high statistics of this measurement result in small experimental uncertainties and smooth behavior of $\operatorname{TKE}\left(A_{H}\right)$.

Fig. 1 includes the average TKE values calculated with FREYA at thermal energies for neutroninduced fission and for spontaneous fission, together with the associated dispersions. Thus the bars 
associated with the FREYA calculations are not sampling errors but indicate the actual width of the TKE distribution for each $A_{H}$.

We assume the average TKE values take the form

$$
\overline{\operatorname{TKE}}\left(A_{H}, E_{\mathrm{n}}\right)=\overline{\operatorname{TKE}}_{\mathrm{data}}\left(A_{H}\right)+d \mathrm{TKE}\left(E_{\mathrm{n}}\right) .
$$

The first term on the right-hand side of Eq. (3) is extracted from the data on the right-hand side of Fig. 1, while the second term is a parameter adjusted to ensure reproduction of the measured average neutron multiplicity, $v$. In a particular fission event, the actual TKE is obtained by adding a thermal fluctuation to the above average.

Once the average total fragment kinetic energy has been obtained, the average combined excitation energy partitioned between the two fragments follows from energy conservation,

$$
\overline{\mathrm{TXE}}=\bar{E}_{L}^{*}+\bar{E}_{H}^{*} \doteq Q_{L H}-\overline{\mathrm{TKE}} .
$$

We assume that the fragment level densities are of the form $\rho_{i}\left(E_{i}^{*}\right) \sim \exp \left(2 \sqrt{ } a_{i} U_{i}\right)$, where $U_{i}$ is the effective statistical energy in the fragment and $a_{i}$ is the level-density parameter [4]. We assume that the asymptotic level density parameter is independent of the fissioning nucleus.

If the two fragments are in mutual thermal equilibrium, $T_{L}=T_{H}$, the total excitation energy will be proportional to the level density parameters, i.e. $\bar{E}_{i}^{*} \sim a_{i}$. FREYA therefore first assigns tentative average excitations based on such an equipartition,

$$
\hat{E}_{i}^{*}=\frac{a_{i}\left(\tilde{E}_{i}^{*}\right)}{a_{L}\left(\tilde{E}_{L}^{*}\right)+a_{H}\left(\tilde{E}_{H}^{*}\right)} \overline{\mathrm{TXE}},
$$

where $\tilde{E}_{i}^{*}=\left(A_{i} / A_{0}\right) \overline{\mathrm{TXE}}$. Subsequently, because the observed neutron multiplicities suggest that the light fragments tends to be disproportionately excited, the average values are adjusted in favor of the light fragment,

$$
\bar{E}_{L}^{*}=x \hat{E}_{L}^{*}, \bar{E}_{H}^{*}=\overline{\mathrm{TKE}}-\bar{E}_{L}^{*},
$$

where $x>1$.

After the mean excitation energies have been assigned, FREYA considers the effect of thermal fluctuations. The fragment temperature $T_{i}$ is obtained from $\bar{U}_{i} \equiv U_{i}\left(\bar{E}_{i}^{*}\right)=a_{i} T_{i}^{2}$. The associated variance in $E_{i}^{*}$ is taken as $\sigma_{i}^{2}=2 \bar{U}_{i}^{*} T_{i}$, where $U\left(E^{*}\right)=E^{*}$ in the simple (unshifted) scenario. Therefore, for each of the two fragments, we sample a thermal energy fluctuation $\delta E_{i}^{*}$ from a Gaussian of variance $\sigma_{i}^{2}$ and modify the fragment excitations accordingly so that

$$
E_{i}^{*}=\bar{E}_{i}^{*}+\delta E_{i}^{*}, i=L, H .
$$

Due to energy conservation, there is a compensating opposite fluctuation in the total kinetic energy [4]. This is accounted for on the right-hand side of Fig. 1.

\section{Neutron Observables}

FREYA assumes that the (fully accelerated) fission fragments first deexcite by (possibly sequential) neutron evaporation, followed by sequential photon emission. At each stage in the decay chain, the spectral shape of the ejectile is determined by the maximum temperature in the daughter nucleus, which in turn is determined by the excitation in the emitting nucleus and the associated $Q$-value. Because there are many different $Q$-values involved, one for each fragment species, and becaue the excitation in the emitter fluctuates, the maximum temperature in the daughter nucleus displays a nontrivial distribution. 


\section{$\mathrm{CNR} * 11$}

The distributions of the maximum temperature in the evaporation daughter, granddaughter, and great granddaughter nuclei are shown on the left-hand side of Fig. 2. The maximum temperature in the daughter nuclei $(v=1)$ is peaked at around $0.5-0.8 \mathrm{MeV}$. The larger contribution comes from the light fragment which tends to be hotter than its heavy partner. The temperature distributions in the granddaughter nuclei $(v=2)$ are considerably broader and peak at lower energies, In the case of the great-granddaughters $(v=3)$ the distributions decrease monotonically. For spontaneous fission of ${ }^{240} \mathrm{Pu}$ where the average total neutron multiplicity is $v \sim 2.15$, it is most likely that each fragment emits just a single neutron so the probability that a fragment emits three neutrons is rather small. While the probability for further neutron emission is typically too small to be visible on the plot, such events do contribute to the overall distribution near $T \sim 0$. The maximum daughter temperature peaks at $T \approx 1 \mathrm{MeV}$ with a tail extending up to $T \approx 2 \mathrm{MeV}$. In addition, the distribution of the maximum temperature in the granddaughter has a distinct peak around $T \approx 0.6 \mathrm{MeV}$, which is not surprising considering that $v \approx 3.75$ for ${ }^{252} \mathrm{Cf}(\mathrm{sf})$.

In the treatment by Madland and Nix [11] a convenient analytical expression was obtained by assuming that the overall distribution of the maximum daughter temperature has a triangular shape. The results in Fig. 2 demonstrate that such an assumption is hardly realistic, even for $v=1$.

The dependence of the average neutron multiplicity on the fragment mass number $A$, is very sensitive to the division of the excitation energy which is governed by the parameter $x$ in Eq. (6). As shown on the right-hand side of Fig. 2, the measurements exhibit a characteristic 'sawtooth' behavior: the neutron multiplicity from the light fragment increases slowly as $A$ approaches $\frac{1}{2} A_{0}$ and then drops rather sharply to a minimum around $A_{H} \sim 130$, the same location as the maximum of $\operatorname{TKE}\left(A_{H}\right)$. Due to the presence of the closed shell at that point, the fragments are particularly resistent to neutron emission. Past the dip region, the multiplicity again increases. The dip tends to be more sharply defined for larger nuclei where $\frac{1}{2} A_{0}$ is close to 130 . For example, the drop is particularly abrupt for ${ }^{252} \mathrm{Cf}$ where $\frac{1}{2} A_{0}=126$. Where data are available, it is seen that the FREYA calculations provide a rather good representation of the 'sawtooth' behavior of $v(A)$, even though FREYA is not tuned to these data.

The left-hand side of Fig. 3 shows the neutron multiplicity distribution $P(v)$. Each emitted neutron reduces the excitation energy in the residue by not only its kinetic energy (recall $\bar{E}=2 T$ where $T$ is the maximum temperature in the daughter nucleus) but also by the separation energy $S_{\mathrm{n}}$ (which is generally significantly larger). Therefore the resulting $P(v)$ is narrower than a Poisson distribution with the same average multiplicity, as clearly seen in the figures.

In experiments, the quantity $P(v)$ is determined by detecting fission events in a sample of material and correlating these with simultaneous neutron detection. The relative probability for emission of $v$ neutrons in given event, $P(v)$, is inferred by combining the calculated probability for observing $n$ neutrons when $v$ were emitted, $Q(n ; v)$, with the detector efficiency determined from the count rate by comparison with a calibration source having a known $v$; typically ${ }^{252} \mathrm{Cf}(\mathrm{sf})$ is used. Thus, while the value of $\bar{v}$ may be well measured for a given isotope, the distribution $P(v)$ is less well determined.

In most cases, the agreement is rather good, with the FREYA results following the data more closely than the equivalent Poisson distribution. The largest difference between the calculated FREYA multiplicity distributions and the data seems to be for ${ }^{240} \mathrm{Pu}(\mathrm{sf})$, which may be due to the smaller sample of ${ }^{240} \mathrm{Pu}(\mathrm{sf}) Y(A)$ and $\operatorname{TKE}\left(A_{H}\right)$ data used as input to FREYA.

For fisison events having a specified total neutron multiplicity $v$, we define the associated spectral shape,

$$
f_{\mathrm{n}}^{v}(E) \equiv \frac{1}{v} \frac{d v}{d E}
$$

which is thus normalized to unity, while the corresponding spectral shape of the neutrons from all the fission events irrespective of the associated multiplicity is denoted simply by $f_{\mathrm{n}}(E)$ and is also normalized to unity.

The multiplicity-gated spectral shapes obtained for the various cases considered are shown on the right-hand side of Fig. 3. Results are presented for multiplicities up to $v=6$. It is apparent that the spectra become progressively softer at higher multiplicities, as one would expect because more neutrons are sharing the available energy. This type of elementary conservation-based correlation feature is not provided by the standard models of fission. The tails of the prompt fission neutron spectra from ${ }^{240} \mathrm{Pu}(\mathrm{sf})$ are longer and broader than those from ${ }^{239} \mathrm{Pu}\left(\mathrm{n}_{\mathrm{th}}, \mathrm{f}\right)$ even though the average energies are 
EPJ Web of Conferences
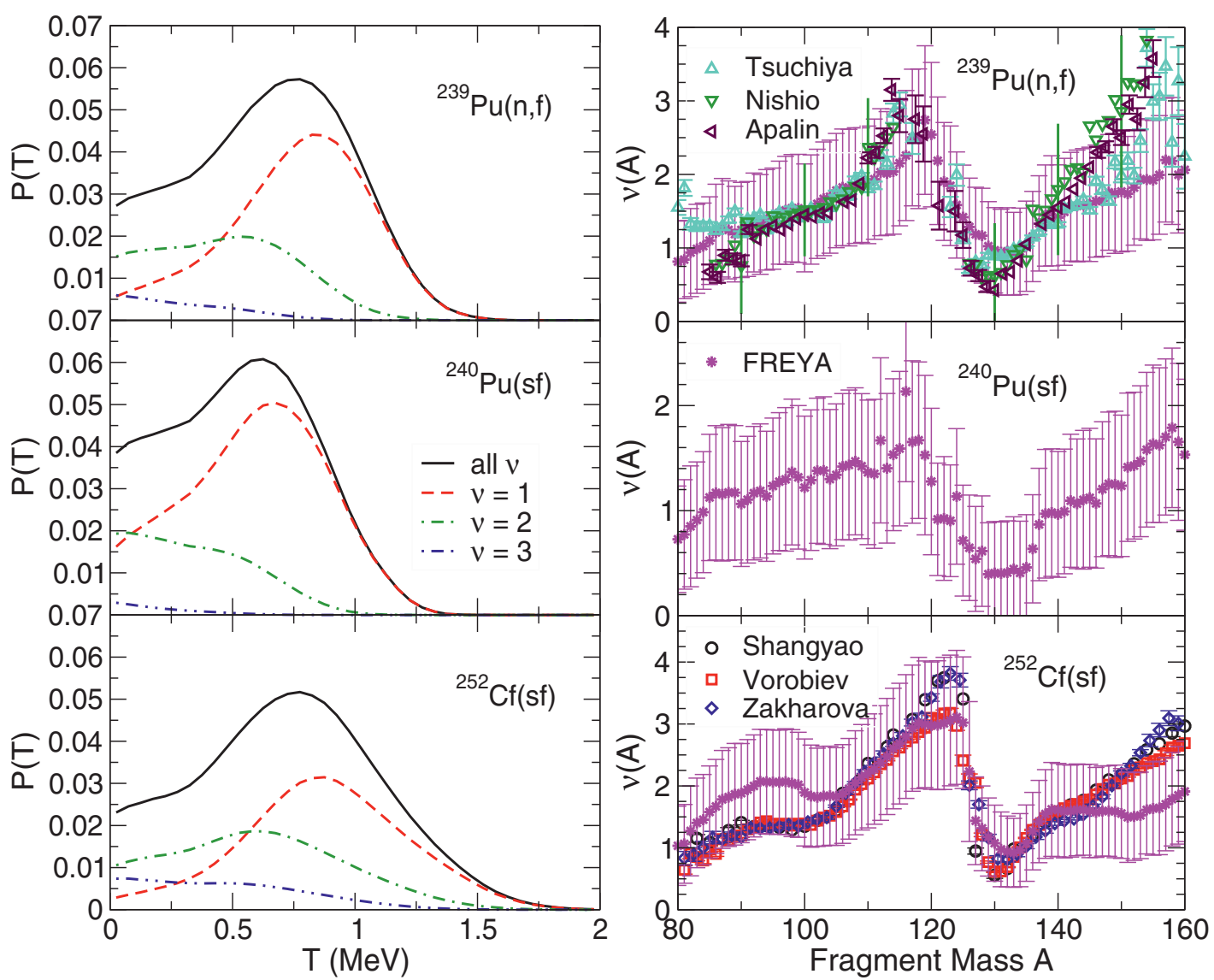

Fig. 2. (Left) The residual temperature distribution for neutron emission as a function of temperature for neutroninduced fission of ${ }^{239} \mathrm{Pu}$ (top) and spontaneous fission of ${ }^{240} \mathrm{Pu}$ (middle) and ${ }^{252} \mathrm{Cf}$ (bottom). The curves show results for $v=1$ (dashed), 2 (dot-dashed), 3 (dot-dot-dashed) and the sum of all neutron emission (solid). (Right) The neutron multiplicity as a function of fragment mass for neutron-induced fission of ${ }^{239} \mathrm{Pu}[12,13,15]$ (top) and spontaneous fission of ${ }^{240} \mathrm{Pu}$ (middle) and ${ }^{252} \mathrm{Cf}$ [16-18] (bottom). The vertical bars at intervals of $A=10$ on the data from Ref. [12] in the top panel are the full-width half maximum of the distribution in neutron multiplicity from the range of charges available for that $A$.The FREYA results also show this variance by the vertical bars. (Note that the scales on the $y$-axes of the plots are not identical.)

smaller and fewer neutrons are emitted. The most energetic neutrons at high multiplicity are emitted from ${ }^{252} \mathrm{Cf}(\mathrm{sf})$ where the spectra are also rather closely clustered around the mean.

The event-by-event nature of FREYA makes it straightforward to extract the angular correlation between two evaporated neutrons which cannot be addressed with the standard models of fission. The left-hand side of Fig. 4 shows this quantity for the neutrons resulting from fission induced by thermal neutrons on ${ }^{239} \mathrm{Pu}$ as well as neutron correlations in spontaneous fission. The results are shown for neutrons with kinetic energies above thresholds at $E=0.5,1$ and $1.5 \mathrm{MeV}$. The angular modulation grows somewhat more pronounced as the threshold is raised albeit with correspondingly reduced statistics.

The neutrons tend to be either forward or backward correlated. The backward correlation appears to be somewhat favored. We have previously analyzed the case of ${ }^{239} \mathrm{Pu}\left(\mathrm{n}_{\mathrm{th}}, \mathrm{f}\right)$ for $v=2$, breaking it down to three separate contributions: both neutrons from the light fragment, both from the heavy fragment, and one neutron emitted from each fragment [24]. There is a significant correlation at $\theta_{12}=0$ when both neutrons are emitted from the same fragment, with a higher peak for the case when both neutrons are emitted from the light fragment due to its higher velocity. On the other hand, when one neutron is emitted from each fragment, their direction tends to be anti-correlated due to the relative motion 

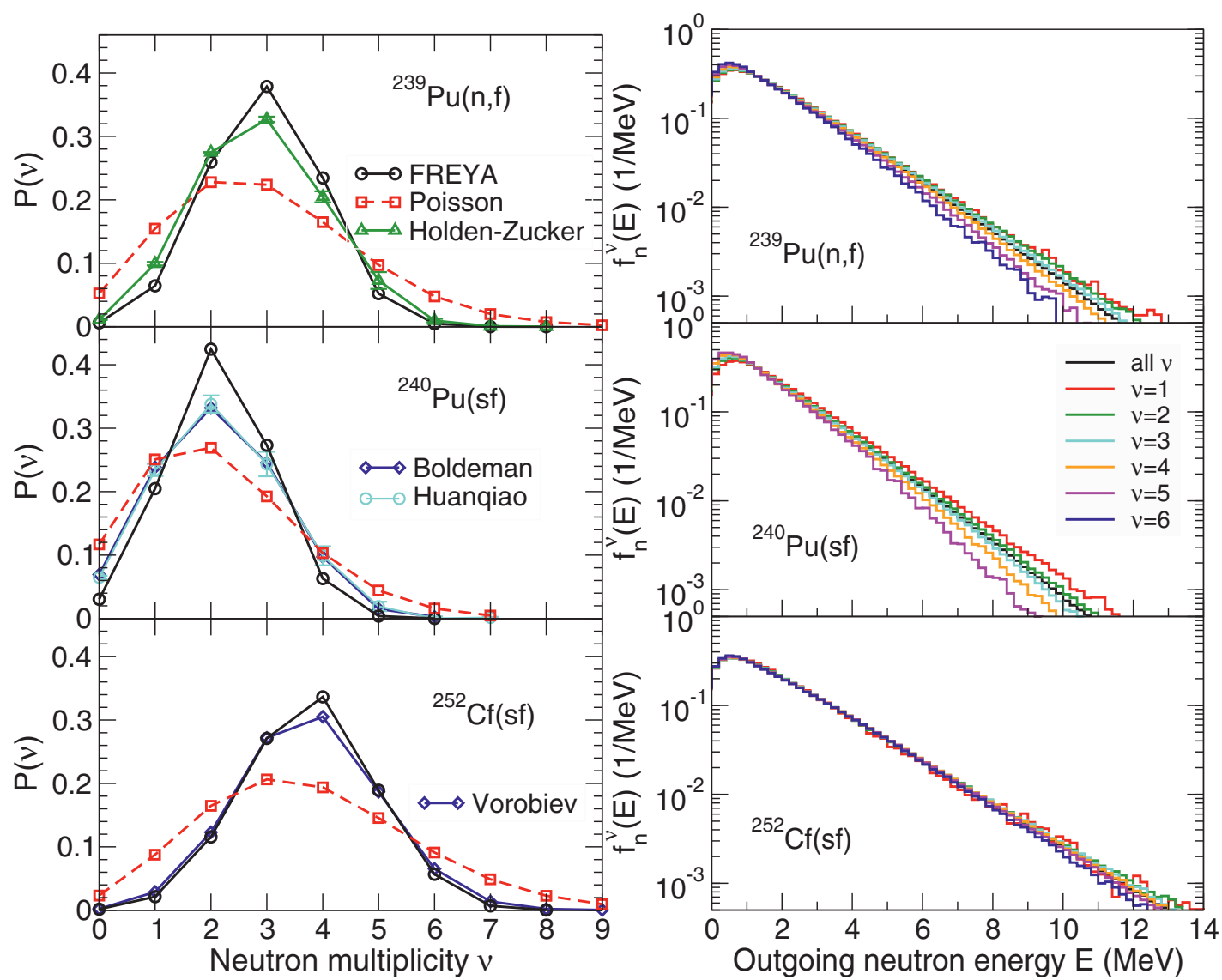

Fig. 3. (Left) The probability for a given neutron multiplicity as a function of multiplicity, $v$, for neutron-induced fission of ${ }^{239} \mathrm{Pu}$ [19] (top) and spontaneous fission of ${ }^{240} \mathrm{Pu}[20,21]$ (middle) and ${ }^{252} \mathrm{Cf}$ [23] (bottom). The FREYA results are shown by the black circles while the equivalent Poisoon distribution is shown by the red squares. (Right) The prompt fission neutron spectrum for neutron induced fission of ${ }^{239} \mathrm{Pu}$ (top) and spontaneous fission of ${ }^{240} \mathrm{Pu}$ (middle) and ${ }^{252} \mathrm{Cf}$ (bottom) as a function of outgoing neutron energy. The FREYA results are shown averaged over all neutron multiplicities as well as the results for neutron multiplicities up to $v=6$.

of the emitting fragments, resulting in a peak at $\theta_{12}=180$. The overall result is a stronger backward correlation because emission from both fragments is most likely. The backward correlation is strongest when the overall neutron multiplicity is low, especially for ${ }^{240} \mathrm{Pu}(\mathrm{sf})$, whereas large multiplicities, as for ${ }^{252} \mathrm{Cf}(\mathrm{sf})$, reduce the angular correlation.

Finally, we discuss correlations between the fission product energies and the neutron multiplicity. The combined kinetic energy of the two resulting (post-evaporation) product nuclei is shown as a function of the neutron multiplicity $v$ in the top panel on the right-hand side of Fig. 4. It decreases with increasing multiplicity, as one might expect since the emission of more neutrons tends to carry off more initial excitation energy, thus leaving less available for the products. As expected from the behavior of $Z_{L} Z_{H}$, the combined product kinetic energy is largest for the most massive fission systems $\left({ }^{252} \mathrm{Cf}\right)$.

The bottom panel on the right-hand side of Fig. 4 show the neutron multiplicity dependence of the average residual excitation energy in those post-evaporation product nuclei. Because energy is available for the subsequent photon emission, one may expect that the resulting photon multiplicity would display a qualitatively similar behavior and thus be anti-correlated with the neutron multiplicity. 

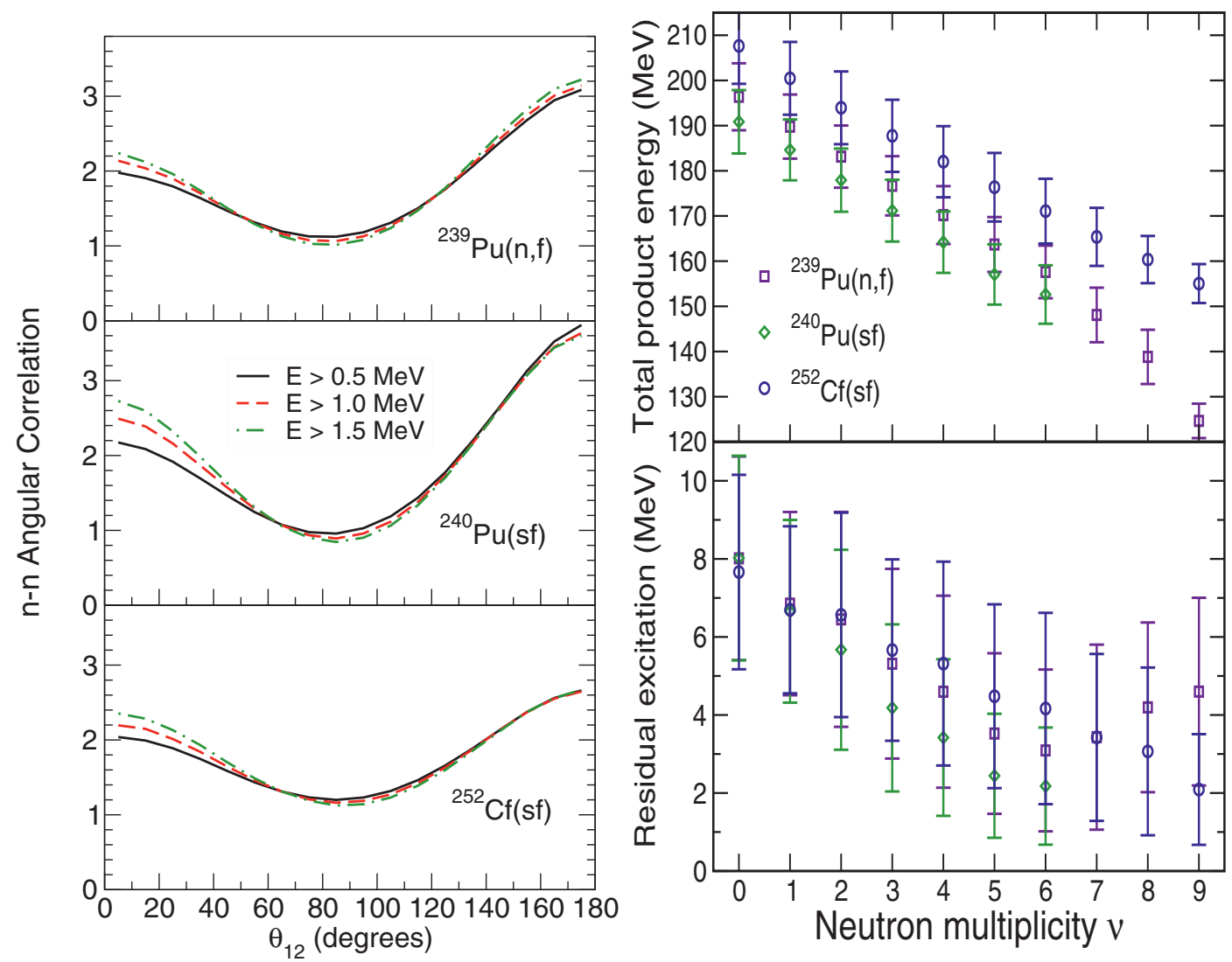

Fig. 4. (Left) The angular correlation between two neutrons emitted during neutron induced fission of ${ }^{239} \mathrm{Pu}$ (top) and spontaneous fission of ${ }^{240} \mathrm{Pu}$ (middle) and ${ }^{252} \mathrm{Cf}$ (bottom) as a function of the opening angle between the two neutrons, $\theta_{12}$. The FREYA results are shown for several cuts on neutron kinetic energy: $E>0.5 \mathrm{MeV}$ (solid black), $1 \mathrm{MeV}$ (dashed red), and $1.5 \mathrm{MeV}$ (dot-dashed green). (Right) The total product kinetic energy (top) and residual excitation energy (bottom) remaining after neutron emission has ceased as a function of neutron multiplicity. The FREYA results are shown for neutron induced fission of ${ }^{239} \mathrm{Pu}$ (squares) and spontaneous fission of ${ }^{240} \mathrm{Pu}$ (diamonds) and ${ }^{252} \mathrm{Cf}$ (circles).

There is little sensitivity of the residual excitation to the identity of the fissioning nucleus. This result suggests that the energy left over after prompt neutron emission is not strongly dependent on the initial fragment temperature.

\section{Concluding remarks}

We have shown that there are characteristic correlations between the emitted neutrons that depend on relative angle, energy and multiplicity. Event-by-event models of fission, such as FREYA, provide a powerful tool for studying fission neutron correlations. Our results demonstrate that these correlations are significant and exhibit a dependence on the fissioning nucleus.

To best take advantage of these correlations, fast response detector systems are desirable. Such systems can better exploit these correlations which would be washed out in slow response detectors and detection systems based on moderators [1]. For experimental groups to better explore the possible correlation studies available with FREYA, we are providing a version to work in-line with several larger Monte Carlo codes, including MCNP [25].

Since our method is phenomenological in nature, good input data are especially important. Some of the measurements employed in FREYA are rather old and statistics limited. It would be useful to repeat 


\section{$\mathrm{CNR} * 11$}

some of these studies with modern detector techniques. In addition, most experiments made to date have not made simultaneous measurements of the fission products and the prompt observables, such as neutrons and photons. Such data, while obviously more challenging to obtain, would be valauble for achieving a more complete understanding of the fission process.

We acknowledge helpful discussions with A. Bernstein and C. Hagmann. The work of R.V. was performed under the auspices of the U.S. Department of Energy by Lawrence Livermore National Laboratory under Contract DE-AC52-07NA27344. The work of R.V. was also supported in part by the National Science Foundation Grant NSF PHY-0555660. The work of J.R. was performed under the auspices of the U.S. Department of Energy by Lawrence Berkeley National Laboratory under Contract DE-AC02-05CH11231. This research is also supported by the US Department of Energy National Nuclear Security Administration Office of Nonproliferation and Verification Research and Development.

\section{References}

1. R. C. Runkle, A. Bernstein and P. E. Vanier, J. Applied Phys. 108, 111101 (2010).

2. R. Vogt, J. Randrup, J. Pruet and W. Younes, Phys. Rev. C 80, 044611 (2009).

3. J. Randrup and R. Vogt, Phys. Rev. C 80, 024601 (2009) [arXiv:0906.1250 [nucl-th]].

4. R. Vogt, J. Randrup, D. A. Brown, M. A. Descalle and W. E. Ormand, submitted to Phys. Rev. C.

5. R. Vogt and J. Randrup, submitted to Phys. Rev. C, arXiv:1109.3788 [nucl-th].

6. S. Lemaire, P. Talou, T. Kawano, M. B. Chadwick, and D. G. Madland, Phys. Rev. C 72, 024601 (2005).

7. W. Younes et al., Phys. Rev. C 64, 054613 (2001).

8. T. R. England and B. F. Rider, LA-UR-94-3106 (1994).

9. P. Schillebeeckx, C. Wagemans, A. J. Deruytter and R. Barthelemy, Nucl. Phys. A 545, 623 (1992).

10. F.-J. Hambsch and S. Oberstedt, Nucl. Phys. A 617, 347 (1997).

11. D. G. Madland and J. R. Nix, Nucl. Sci. Eng. 81, 213 (1982).

12. K. Nishio, Y. Nakagome, I. Kanno, and I. Kimura, J. Nucl. Sci. Technol. 32, 404 (1995).

13. C. Tsuchiya, Y. Nakagome, H. Yamana, H. Moriyama, K. Nishio, I. Kanno, K. Shin, and I. Kimura, J. Nucl. Sci. Technol. 37, 941 (2000).

14. C. Wagemans, E. Allaert, A. Deruytter, R. Barthélémy, and P. Schillebeeckx, Phys. Rev. C 30, 218 (1984).

15. V. F Apalin, Yu. N. Gritsyuk, I. E. Kutikov, V. I. Lebedev, and L. A. Mikaelian, Nucl. Phys. A 71, $553(1965)$.

16. D. Shengyao, X. Jincheng, L. Zuhua, L. Shaoming, and Z. Huanqiao, Chin. Phys. 4, 649 (1984).

17. V. N. Dushin, F. J. Hambsch, V. A. Yakovlev, V. A. Kalinin, I. S. Kraev, A. B. Laptev, D. V. Nikolav, B. F. Petrov, G. A. Petrov, V. I. Petrova, Y. S. Pleva, O. A. Shcherbakov, V. I. Shpakov, V.

E. Sokolov, A. S. Vorobiev, T. A. Zavarukhina, Nucl. Instrum. Meth. A 516, 539 (2004).

18. V. P. Zakharova, D. K. Ryazanov, B. G. Basova, A. D. Rabinovich, V. A. Korostylev, Sov. J. Nucl. Phys., 30, 19 (1979).

19. N. E. Holden and M. S. Zucker, "A Reevaluation of the Average Prompt Neutron Emission Multiplicity ( v) Values from Fission of Uranium and Transuranium Nuclides", BNL-NCS-35513, Brookhaven National Laboratory (1985).

20. Z. Huanqiao, L. Zuhua, D. Shengyao and L. Shaoming, Nucl. Sci. Eng. 86, 315 (1984).

21. J. W. Boldeman, Second Conference on Neutron Physics, 4, 114 (1973);

http://www-nds.iaea.org/exfor/servlet/X4sGetSubent?subID=30376006.

22. H. R. Bowman, J. C. D. Milton, S. G. Thompson, and W. J. Swiatecki, Phys. Rev. 126, 2120 (1962); Phys. Rev. 129, 2133 (1963).

23. A. S. Vorobiev, V. N. Dushin, F. J. Hambsch, V. A. Yakovlev, V. A. Kalinin, I. S. Kraev, A. B. Laptev, B. F. Petrov, G. A. Petrov, Yu. S. Pleva, O. A. Shcherbakov and V. E. Sokolov, http://www-nds.iaea.org/exfor/servlet/X4sGetSubent?subID=41425004.

24. J. Randrup and R. Vogt, JRC Scientific and Technical Reports 64789, 115 (2011).

25. F. B. Brown et al., Trans. Am. Nucl. Soc. 87, 273 (2002). 